\title{
SPATIALLY NON-HOMOGENEOUS COAGULATION EQUATIONS WITH SOURCE TERMS
}

\author{
BY \\ M. SHIRVANI AND H. J. VAN ROESSEL \\ Department of Mathematical and Statistical Sciences, University of Alberta, Edmonton, Alberta, \\ Canada
}

\begin{abstract}
The physical process of coagulation or coalescence of particles is often modelled by Smoluchowski's coagulation equation, an infinite system of nonlinear differential equations governing the binary interactions of particles of different sizes. One of the physical assumptions underlying the coagulation equation is spatial homogeneity, in particular, the assumption that coagulation of particles is governed only by particle type or size. The physical shape of the cloud of particles, as well as the relative position of particles of various type, are ignored.

One way to model spatial inhomogeneities is to consider several localized clusters of particles distributed within the cloud, with each cluster being a microcosm of coagulating particles. That is to say that coagulation occurs among particles within a cluster but not among particles from different clusters. Interaction between clusters is then accommodated by allowing for a migration, or drift, of particles between clusters.

In this article, we model this cloud of localized clusters by a directed graph, with a vertex located at each cluster of particles and a directed edge between vertices representing the migration of particles between clusters, and investigate the effect of the shape of the graph on the properties of the solutions and their evolution.

For the case of a constant coagulation kernel, we prove the existence, uniqueness, and global stability of the solutions, as well as mass conservation, in this more general setting. We also give examples of some of the novel features arising from the structure of the graph, such as the possibility of oscillatory behavior, and the much greater degree of control over the creation of large particles, a problem of some importance in practical applications.
\end{abstract}

Received April 15, 2003.

2000 Mathematics Subject Classification. Primary 82D60.

Research was supported by the Natural Sciences and Engineering Research Council of Canada. 
1. Introduction. In a previous paper [7] we proved the existence and the uniqueness of the solutions to the coagulation system

$$
x_{k}^{\prime}=\frac{1}{2} \sum_{i+j=k} x_{i} x_{j}-x_{k} \sum_{i=1}^{x} x_{i}+s_{k} .
$$

for all $t \geqslant 0$ under the assmmptions that. for all $k$, we have $s_{k} \geqslant 0$ for all $t$ with $S(t)=\sum_{i=1}^{x} s_{i}(t)$ locally integrable. $x_{k}(0) \geqslant 0$. and $\sum_{i=1}^{x} x_{i}(0)<x$. It was also shown that if the limits (as $t \rightarrow \infty$ ) of $S$ and the $s_{k}$ exist. then so do all the limits $x_{k}(x)$. and we provided a recursive formula for the latter limits. We also looked at the practical problem of controlling the number of particles of various sizes.

One of the physical assumptions underlying the derivation of (1) is spatial homogeneity, requiring, in particular. the assimption that every particle of type $i$ has an equal probability of interacting with every particle of type $j$. The physical shape of the cloud of particles, as well as factors such as spatial drift. are ignored. (For numerical work and discussion of some of the relevant features. see for example [2]. [4], and [5].)

In this article, we model drift and spatial factors (such as the shape of the vessel containing the particles) by using a finite directed graph $\Gamma$ as an approximation to the shape of the particle cloud. Each vertex, of the graph represents a (small) region of the cloud, where a system of coagulating particles resides. Particles from different vertices do not coagulate with each other. Instead. particles drift from site to site: If $\alpha \beta$ is a directed edge in $\Gamma$, then particles at site $\alpha$ drift into site $\beta$ at a rate proportional to their number. The classical case may be regarded as that of a graph with a single vertex and no edges.

This model exhibits a number of interesting new features such as possible oscillatory behaviour (with oscillations passing from vertex to vertex) and much greater control over the number of particles created. It may be conjectured that many practical problems have graphs over which they evolve in a maximal fashion (we give examples of this in Sec. 2).

The obvious generalization is the following:

$x_{\alpha, k}^{\prime}=\frac{1}{2} \sum_{i+j=k} K_{i, j} x_{\alpha, i} x_{\alpha, j}-x_{\alpha, k} \sum_{i=1}^{\infty} K_{i, k} x_{\alpha, i}+\sum_{\beta} g_{\beta \alpha, k} \cdot x_{\beta, k}-\sum_{\beta} g_{\alpha \beta, k} x_{\alpha, k}+s_{\alpha, k}$.

for all $k$ and all $\alpha \in V$, the set of vertices of $\Gamma$. where $s_{\alpha . k} \geqslant 0$ are input terms and $K_{i . j}=K_{j . i}$ are the coagulation kernels. A term $g_{\alpha \beta . k} \cdot x_{\alpha . k}$ indicates that $\alpha \beta$ is an edge from $\alpha$ to $\beta$, and that $g_{\alpha \beta, k}$ is the positive constant of proportionality of the rate of drift of particles of type $k$ from site $\alpha$ to site $\beta$. With this interpretation, we must assume that $\sum_{\alpha} g_{\alpha \beta} \leqslant 1$ for all $\beta$.

In order to observe the influence of the structure of the graph on the evolution of the system. we confine ourselves to the case where the systems at each vertex coagulate according to (1), this being a case where one has a thorough understanding of the singlevertex system. To make calculations more manageable, assume further that $g_{\alpha \beta, k}=g_{\alpha, 3}$ is independent of $k$ for all $\alpha, \beta \in V$. the vertex set of $\Gamma$. The system considered in this 
paper is, therefore.

$$
x_{\alpha, k}^{\prime}=\frac{1}{2} \sum_{i+j=k} x_{\alpha, i} x_{\alpha, j}-x_{\alpha, k} \sum_{i=1}^{\infty} x_{\alpha, i}+\sum_{3} g_{3 \alpha} \cdot r_{3 . k}-\sum_{3} g_{\alpha, 3} x_{\alpha . k}+s_{\alpha, k} .
$$

subject to the conditions that for all $\alpha \in V, k \geqslant 1$, and $t \geqslant 0$, we have $s_{\alpha . k}(t) \geqslant 0$, $x_{\alpha, k}(0) \geqslant 0, x_{\alpha}(0)=\sum_{k=1}^{\infty} x_{\alpha, k}(0)<\infty$, with the $s_{\alpha . k}$ and $s_{\alpha}=\sum_{k=1}^{\infty} s_{\alpha . k}$ locally integrable.

The paper is organized as follows: A number of examples of the novel features of the model are presented in Sec. 2. Some graph-theoretical and matrix preliminaries and definitions are collected in Sec. 3. The next two sections deal with the existence and the uniqueness of the solutions to (2). Not unexpectedly, (2) has a unique non-negative solution for all time, although the multi-dimensional nature of the problem makes the proofs considerably more intricate than the familiar single-vertex system. The global stability of the solutions is investigated in Sec. 6. Under the assumption that the $s_{a . k}$ and $s_{\alpha}$ all have limits as $t \rightarrow \infty$, we show that (2) has either no equilibrium points or a global attractor in the positive octant. We also provide recursive formulae for the limits, when they exist. Conservation of mass is investigated in Sec. 7. It is shown that, if all the $s_{\alpha, k}=0$, then the total mass $\sum_{\alpha, k} k x_{\alpha . k}$ of the system is conserved for all time, although the total mass $\sum_{k} k x_{\alpha, k}$ at each vertex does vary with time. and can indeed have a damped oscillatory evolution.

We proceed as follows. Write $x_{\alpha}=\sum_{i=1}^{\infty} x_{\alpha, i}$, and $s_{\alpha}=\sum_{i=1}^{\infty} s_{\alpha, i}$. Formal summation of (2) over all $k$ yields the system of ODEs

$$
x_{\alpha}^{\prime}=-\frac{1}{2} x_{\alpha}^{2}+\sum_{\beta} g_{\beta \alpha} x_{\beta}-\sum_{\beta} g_{\alpha, 3} x_{\alpha}+s_{\alpha}
$$

for all $\alpha \in V$, subject to the initial conditions $x_{\alpha}(0)=\sum_{i=1}^{\infty} x_{\alpha, i}(0)<\infty$. Corresponding to (2) we have the system

$$
y_{\alpha, k}^{\prime}=\frac{1}{2} \sum_{i+j=k} y_{\alpha, i} y_{\alpha, j}-x_{\alpha} y_{\alpha, k}+\sum_{\beta} g_{i \beta \alpha} y_{\beta, k}-\sum_{\beta} g_{\alpha \beta} y_{\alpha, k}+s_{\alpha, k}
$$

obtained by replacing the infinite sum $\sum_{i=1}^{\infty} x_{\alpha . i}$ in (2) by the solution $x_{\alpha}$ of (3). The point, as usual, is that the presence of the infinite sum in (2) means that there is no $a$ priori reason why (2) would be well-defined, whereas (3) and (4) always are. We begin by proving that the solutions to (4) satisfy $\sum_{i=1}^{\infty} y_{\alpha, i}=x_{\alpha}$. Thus, the $y_{\alpha, i}$ represent a set of solutions to (2).

We use the following convention for naming objects: Suppose we have scalars $f_{\alpha . k}$ for all $\alpha \in V$ and all $k=1,2, \ldots$. We then write $f_{\alpha}$ for $\sum_{k=1}^{\infty} f_{\alpha, k}$, and $f_{k}$ for the vector $\left(f_{\alpha, k}\right)_{\alpha \in V}$ whose components are indexed by the vertices $\alpha$ of $\Gamma$. As lower-case greek letters are used exclusively for the vertices of graphs. this should cause no confusion.

2. Examples. A first noteworthy feature of the new set-up is the possibility of (damped) oscillatory behaviour at each vertex (even with constant input functions). Let $\mu_{\alpha}(t)=\sum_{k=1}^{\infty} k x_{\alpha, k}(t)$ for all $\alpha$, set $\underline{\mu}=\left(\mu_{\alpha}\right)$, and assume that all the source terms 
$s_{\alpha, k}=0$. From Sec. 7, we know that

$$
\underline{\mu}(t)=e^{\mathrm{H} t} \underline{\mu}(0)
$$

for all $t$, where $\mathrm{H}$ is the matrix with entries labelled by the vertices of $\mathrm{I}$ such that $H_{\alpha, \alpha}=-\sum_{\beta} g_{\alpha \beta \beta}$ and $H_{\alpha, 3}=g_{\beta \alpha}$ for all $\alpha \neq \beta$. Noreover, the total mass of the system is conserved for all time, as in the single-vertex case. On the other hand. if $\mathrm{H}$ has non-real eigenvalues, then (5) shows that the individual components of $\underline{\mu}(t)$ can have damped oscillatory behaviour. The simplest example is the graph $\mathcal{P}_{3 b}$ in Fig. 1, with $g_{1,2}=g_{2.3}=g_{3.1}=g>0$. The eigenvalues are easily seen to be the three cube roots of $g$, two of which are, of course, complex.
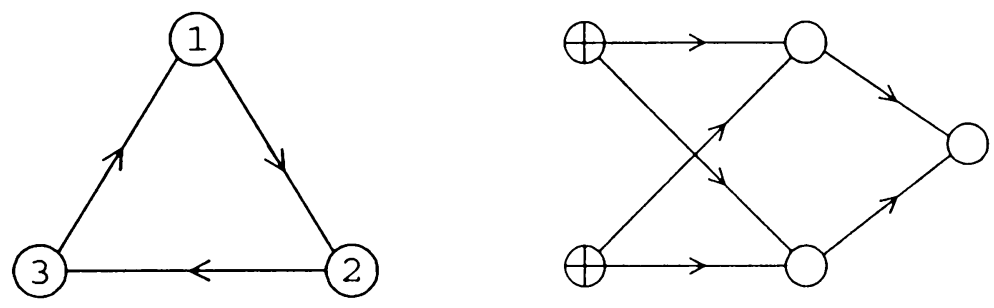

FIc. 1. Graphs $\mathcal{P}_{3 b}$ and $\mathcal{P}_{5 a}$.

One practical application of the new model is to the problem of controlling the number of large and small particles created by the coagulation process. It will be shown below (Sec. 6) that the limiting values $\ell_{\alpha}=x_{\alpha}(\infty)$ and $\ell_{\alpha, k}=x_{\alpha . k}(\infty)$ exist if the input functions $s_{\alpha}$ and $s_{\alpha, k}$ have limits as $t \rightarrow \infty$. Specifically, let us write $\lambda=\sum_{\alpha} x_{\alpha}(\infty)$ for the limiting total number of particles in the system, $\rho_{n}=\left(\sum_{\alpha x} \sum_{k \geq n} x_{\alpha, k}(\infty)\right) / \lambda$ for the limiting percentage of the total number of particles of types $k \geqslant n$, and $\rho_{n}^{*}=\rho_{n} / v$ for the average percentage of large particles per vertex (here $v$ is the number of vertices of $\Gamma)$. For the single-vertex case, the formulae in Theorem 4.4 of [7] allow us to calculate the $\rho_{n}=\rho_{n}^{*}$ recursively.

To take a concrete example, assume that the entire input into the system consists of particles of type 1 , and consider $\rho_{4}$ (the qualitative conclusions remain the same for larger values of $n$ ). In the single-vertex case, we have $\rho_{4}^{*}=\rho_{4}=0.3125$, regardless of the total input into the system (as long as the input is greater than zero).

In the multi-vertex case. the corresponding numbers of course depend on both the shape of the graph and the value of the drift coefficients $g_{\alpha \beta}$. so we only give a few examples to indicate the range of phenomena encountered. In all cases the total input was 1 unit, consisting entirely of particles of type 1, and divided equally between the initial vertices of the graph (those with only outgoing edges) if there were any in the graph. The values of the $g_{\alpha \beta}$ were either set at the moderately small value of 0.2 , or at their maximum, so $g_{\alpha \beta}$ was constant at each $\alpha$ and added up to 1 (experiments show that this is a particularly efficient choice).

For the graphs depicted in Figs. 1, 2, and 3, the extreme values for $\rho_{4}$ and $\rho_{4}^{*}$ are listed in Table 2 (the complete set of data can be found in Sec. 8): 


\begin{tabular}{|l||l|l|l|l|}
\hline & $\min \rho_{4}$ & $\max \rho_{4}$ & $\min \rho_{4}^{*}$ & $\max \rho_{4}^{*}$ \\
\hline \hline$g=g_{\max }$ & 0.156 for $\mathcal{H}_{4,2}$ & 0.324 for $\mathcal{P}_{3 b}$ & 0.017 for $\mathcal{H}_{4,2}$ & 0.126 for $\mathcal{A}_{2}$ \\
\hline$g=0.2$ & 0.286 for $\mathcal{P}_{5 a}$ & 0.417 for $A_{6}$ & 0.030 for $\mathcal{H}_{4,2}$ & 0.169 for $\mathcal{A}_{2}$ \\
\hline
\end{tabular}

Table 2: Extreme values of $\rho_{4}$ and $\rho_{4}^{*}$.
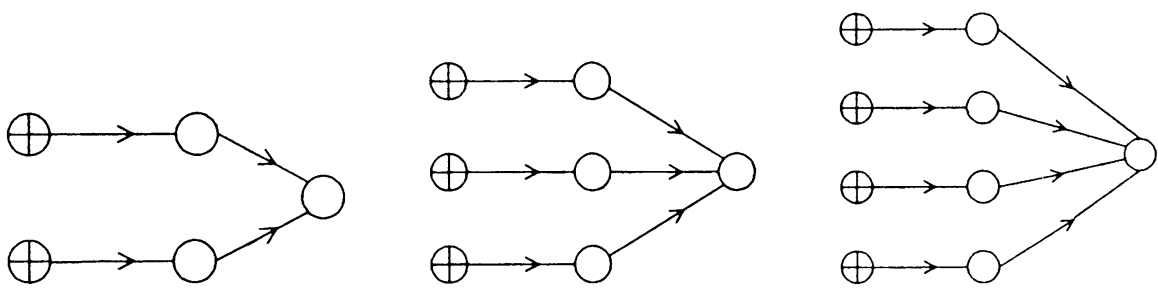

FIC. 2. Graphs $\mathcal{H}_{2,2}, \mathcal{H}_{3,2}$, and $\mathcal{H}_{4,2}$.

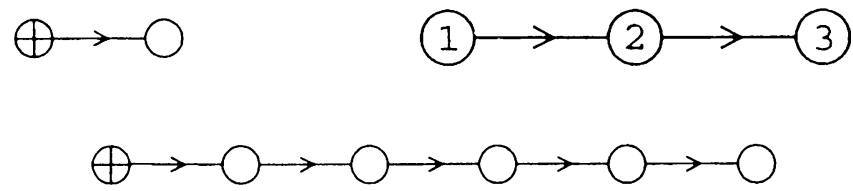

FIG. 3. Graphs $\mathcal{A}_{2}, \mathcal{A}_{3}$, and $\mathcal{A}_{6}$.

However, $\rho_{4}^{*}$ does not decrease simply because the graph has more vertices. The graph $\mathcal{A}_{6}$ has a higher average number of large particles per vertex than several graphs on five vertices, including $\mathcal{H}_{2,2}$. The graph $\mathcal{H}_{4,2}$ is particularly efficient for reducing the number of large particles.

The above examples suggest the following problem. Consider a fixed graph $\Gamma$ and fixed input vectors $s_{k}(t)=m_{k}$. The $\ell_{\alpha}$ and the $\ell_{k}$ are continuous functions of the parameters $g_{\alpha \beta} \in[0,1]$ and hence so are the $\rho_{n}$. But the $g_{\alpha \beta}$ range over the unit cube, which is compact, so $\rho_{n}$ has an absolute maximum and an absolute minimum. These values (and the corresponding values of the parameters) are, therefore, absolute invariants of the graph $\Gamma$ for the given input vectors, and measure its efficiency in producing large particles. They are, however, almost impossible to obtain analytically in a useful form. What can be said in general is that, as all the $g_{\alpha \beta} \rightarrow 0$, the graph approximates more and more to a collection of disconnected vertices, each of which will produce the same values of $\rho_{n}$ and $\rho_{n}^{*}$ as in the single-vertex case.

For example, consider $\Gamma=\mathcal{A}_{2}$. Figure 4 , which is a plot of $\rho_{4}$ as a function of $g=g_{\alpha \beta}$, indicates an absolute maximum of about 0.352 at $g=0.065$ and an absolute minimum of 0.252 at $g=1$ (for an input of one unit at the vertex marked with a + ):

For $\Gamma=\mathcal{A}_{3}$, things are more interesting. Numerical calculations indicate an absolute maximum of around 0.378 when $\left(g_{1,2}, g_{2.3}\right)=(0.085,0.061)$, and an absolute minimum of around 0.229 when $\left(g_{1,2}, g_{2.3}\right)=(1,0.384)$.

As our final example, we consider the connection between the standard single-vertex model and the more general models of this paper. As an $n$-vertex analogue, let $\Gamma=\Gamma_{n}$ be 


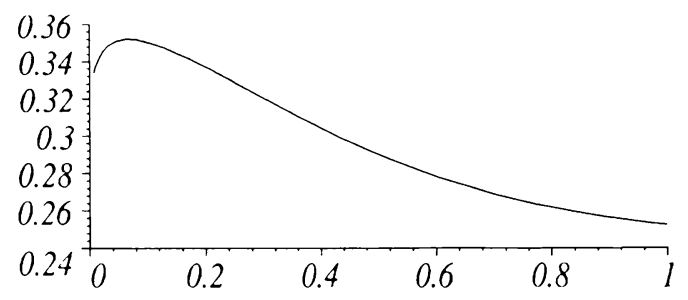

Fici. 4. Plot of $\rho_{4}(g)$ for the graph $\mathcal{A}_{2}$.

the complete directed graph on $n$ vertices. so we have an edge a.3 for every pair of distinct vertices $\alpha$ and $\beta$. Fix $g \in(0.1)$ and define $g_{\alpha ;}=g$ for all $\alpha$ and $\beta$. Brief experimentation shows that the various $x_{a}$ and $x_{a, k}$ are not scaled versions of the corresponding functions in the single-vertex (ase. no matter how the source terms and initial values are defined.

The situation. however. is quite different for the limiting distributions. Assume that the single-vertex input is distributed equally among the vertices of $\Gamma$. If $s_{k}$ and $s=$ $\sum_{k=1}^{x} s_{k}$ are source functions in the single-vertex case. then clearly $s_{\alpha}(\infty)=s(\infty) / n$ and $\mu_{k}=s_{\alpha, k}(\infty)=s_{k}(x) / n$ for all $\alpha$. The various quantities being identical at every vertex, it is clear that every automorphism $a \mapsto a^{*}$ of the graph $\Gamma$ maps $x_{a . k}$ into $x_{a \cdot k}$ and $x_{\alpha}$ into $x_{\alpha^{*}}$. Thuss, the values of $\ell_{n}=x_{n}(\infty)$ and $\ell_{\alpha . k}=x_{\alpha . k}(\infty)$ are independent of $a$. Equation (21) therefore loads to

$$
\ell_{0}=\sqrt{\frac{2 . s(x)}{n}}
$$

for all $a$. As for the (a.k. simmetry implies that $t_{k}=\lambda_{k} \cdot 1$. The matrix $\mathrm{P}$ of $(30)$ has cutries $P_{\alpha, \alpha}=\sqrt{2 s(\infty) / n}+(n-1) g$ and $P_{\alpha . \beta}=-g$ for all $\alpha$ and $\beta$. Therefore. $\mathrm{P} \underline{1}=\sqrt{2 s(x) / n} \underline{1}$. Equation (29) readily reduces to

$$
\sqrt{\frac{2 s(x)}{n}} \lambda_{k i} \underline{1}=\left(\frac{1}{2} \sum_{i+j=k} \lambda_{i} \lambda_{j}+\frac{s_{k}(\infty)}{n}\right) \underline{1} .
$$

The generating functions $F_{n}(\zeta)=\sum_{k=1}^{x} \lambda_{k} \cdot \zeta^{k}$ and $C_{\boldsymbol{r}}(\zeta)=\sum_{k=1}^{x} \mu_{k} \cdot \zeta^{\zeta^{k}}$ then satisfy

$$
\sqrt{\frac{2 s(x)}{n}} F_{n}=\frac{1}{2} F_{n}^{2}+\frac{1}{2 n} G \text {. }
$$

Now $F_{1}$ is the generating function of the single-vertex case. and clearly the above equation has the solution $F_{n}=n^{-1 / 2} F_{1}$. This shows that the limiting distribution of the singlevertex case is a scalar multiple of the limiting distribution of the particular $n$-vertex problem defined above. eren though the evolution functions are not related in any simple way. (The reader may verify that the same conclusion holds if, instead of the complete graph on $n$ vertices, we had taken the graph on $n$ vertices where for some $r$ between 1 and $n-1$, each vertex has $r$ edges going in and $r$ edges going out.)

3. Notation and Preliminary Results. We consider finite directed graphs without loops (= edges beginning and ending at the same vertex), although double edges ( $\alpha .3$ and $\beta \alpha)$ are allowed. A (directed) path $p$ in $\Gamma$ is a secpuence $\left(\alpha_{1} \ldots \ldots \alpha_{n}\right)$ of $n \geqslant 2$ vertices 
such that $\alpha_{i} \alpha_{i+1}$ is an edge in $\Gamma$ for $i=1, \ldots, n-1$. For $p$ as above, write $o(p)=\alpha_{1}$, $t(p)=\alpha_{n}$, and $\ell(p)=n-1$. In the context of (2), set $g_{p}=\prod g_{e}$ where the product is taken over all the edges $e=\alpha_{i} \alpha_{i+1}$ of $p$. A path $p$ is a cycle if $t(p)=o(p)$, and $p$ is simple if no vertex is repeated in $p$ (except possibly $t(p)=o(p)$ ). Of course, $\Gamma$ only has a finite number of simple paths.

Any path $p$ may be regarded as consisting of a number of simple cycles attached to a simple path at various vertices (usually in a non-unique fashion): If $\ell(p)=1$, then $p=\beta \alpha$ is an edge and $\beta \neq \alpha$, so $p$ is simple. When $\ell(p)>1$ and $p$ is not simple, let $p=\ldots \alpha \ldots \alpha \ldots$ be an occurrence of a repeated vertex, chosen so that no other vertex of $p$ is repeated between the indicated $\alpha$ s. Then the part of $p$ between these occurrences of $\alpha$ is a simple cycle $C$, and the segments of $p$ preceding and following the two $\alpha$ s can be attached at $\alpha$ to form a shorter path $q$. By induction on the length of the path, $q$ is formed by attaching simple cycles to a simple path, and $p$ is obtained by attaching $C$ to $q$ at $\alpha$.

The above argument shows that for any path $p$, we have $g_{p}=\prod_{C} g_{C} g_{p_{j}}^{\varepsilon}$, where the first factor is a possibly empty product over certain simple cycles, $p_{j}$ is a non-cyclic simple path, and $\varepsilon=0,1$. Similarly, $\ell(p)=\sum_{C} \ell(C)+\varepsilon \ell\left(p_{j}\right)$.

The structure of the system (2) depends greatly on the subgraph structure of $\Gamma$. A subgraph $\Delta$ of $\Gamma$ is said to be legitimate if, for every $\alpha \in V(\Delta)$, every path $p$ in $\Gamma$ that ends in $\alpha$ lies entirely in $\Delta$. Every vertex $\alpha$ of $\Gamma$ lies in a smallest legitimate subgraph ${ }_{\alpha} \Gamma$ of $\Gamma$, namely, the union of all the paths in $\Gamma$ that terminate in $\alpha$. If $\Delta$ is a legitimate subgraph of $\Gamma$, then by definition no edge of $\Gamma$ leads into $\Delta$, and the subsystem of (2) indexed by the vertices in $\Delta$ essentially decouples from the rest of (2). Clearly, $\Gamma$ has no proper legitimate subgraphs if and only if $\Gamma={ }_{\alpha} \Gamma$ for all vertices $\alpha$, which happens if and only if there is a path between any two vertices of $\Gamma$.

There are two matrices associated to Eq. (2). The first is $G=\left(g_{\alpha \beta}\right)$, which has the property that $c_{\alpha}=(\mathrm{G} \underline{1})_{\alpha}=\sum_{\beta} g_{\alpha \beta} \leqslant 1$, where $\underline{1}$ indicates the (row or column) vector consisting of $1 \mathrm{~s}$. The second matrix (yielding the linear term in (2)) is $\mathbf{H}=$ $-\operatorname{diag}\left(c_{\alpha}\right)+\mathrm{G}^{T}$, so $H_{\alpha, \alpha}=-c_{\alpha}$ and $H_{\alpha, \beta}=g_{\beta \alpha}$ for all $\alpha \neq \beta$. Plainly, therefore, $\underline{1} \mathrm{H}=0$.

Recall that a matrix $\mathrm{H}$ (relative to a fixed basis) is said to be reducible if the basis can be re-ordered into $\left\{u_{1}, \ldots, u_{m}, v_{1}, \ldots, v_{n}: m, n \geqslant 1\right\}$ such that every $\mathrm{H} u_{i}$ is a linear combination of the $u_{1}, \ldots, u_{m}$ only, i.e., a permutation of the basis relative to which the matrix of $\mathrm{H}$ has block upper triangular form. If no such permutation exists, then $\mathrm{H}$ is said to be irreducible.

We also need to use a particular partial order on the set of vectors indexed by $V$. Given vectors $\underline{a}=\left(a_{\alpha}\right)$ and $\underline{b}=\left(b_{\alpha}\right)$, write $\underline{a} \leqslant \underline{b}$ if and only if $a_{\alpha} \leqslant b_{\alpha}$ for every $\alpha$. This is only a partial order (e.g., $(1,-1)$ and $(-1,1)$ are incomparable). We also write $\underline{a}<\underline{b}$ if and only if $a_{\alpha}<b_{\alpha}$ for every $\alpha$.

Lemma 3.1. Let $\boldsymbol{H}$ be the matrix defined above for (2).

(i) 0 is an eigenvalue of $\mathrm{H}$ with at least one eigenvector $\underline{u} \geqslant 0$, and every eigenvalue of $\mathrm{H}$ has non-positive real part. 
(ii) If $\mathrm{H}$ is irreducible, then 0 is a simple eigenvalue, $\underline{u}>0$, and every other eigenvalue of $\mathrm{H}$ has negative real part.

(iii) $\mathrm{H}$ is irreducible if and only if there is a directed path between any two vertices of $\Gamma$.

Proof. The matrix $\mathrm{I}+\mathrm{H}$ is a stochastic matrix because it has non-negative entries and all of its column sums are equal to 1 . As is well known. its spectral radius is then 1 , and it has 1 as an eigenvalue with an eigenvector $\underline{u} \geqslant 0$ (see [1], XIII. Sec. 6). Next. it is obvious that $\mathrm{I}+\mathrm{H}$ is irreducible if and only if $\mathrm{H}$ is. An irreducible stochastic matrix is known to have 1 as an eigenvalue of multiplicity 1 with an eigenvector $\underline{u}>0$. This proves (i) and (ii).

We turn to the proof of (iii). Suppose $\mathrm{H}$ is reducible, and let $V_{1}$ and $V_{2}$ denote the disjoint index sets corresponding to the permuted basis which puts $\mathrm{H}$ into block triangular form. If $\alpha \in V_{1}$ and $\beta \in V_{2}$, then by definition $H_{3 \alpha}=0$ : i.e. $g_{\alpha \beta}=0$, whence $\alpha, 3$ is not an edge in $\Gamma$. Let $\Gamma_{i}$ be the subgraph of $\Gamma$ consisting of the vertices in $V_{i}$ and those edges $e$ of $\Gamma$ such that $o(e) . t(e) \in V_{i}$. The above argument shows that there is no edge from $\Gamma_{1}$ to $\Gamma_{2}$. In particular. if $\beta \in \Gamma_{2}$, then ${ }_{3} \Gamma \subseteq \Gamma_{2}$. which means that $\Gamma_{2}$ is a proper legitimate subgraph of $\Gamma$. Conversely. if $\Delta$ is a proper legitimate subgraph of $\Gamma$, then $V(\Delta)$ and $V \backslash V(\Delta)$ yield a partition of the basis relative to which $\mathrm{H}$ is seen to be block triangular. The upshot is that $\mathrm{H}$ is irreducible if and only if $\Gamma$ has no proper legitimate subgraphs, which proves (iii).

We also need the following theorem on Minkowski matrices (see, e.g., [8], Theorem 1. Sec. 11):

Theorem 3.2. Let $\mathrm{T}=\left(T_{i, j}\right)$ be a real $n \times n$ matrix such that $T_{i, j} \leqslant 0$ for all $i \neq j$. Then the following are equivalent:

(1) $T^{-1}$ exists and has non-negative entries.

(2) There exists a vector $\underline{v}>\underline{0}$ such that $T \underline{v}>\underline{0}$.

(3) Every eigenvalue of $\mathrm{T}$ has positive real part.

4. Existence. The first step is to prove that the solutions to (3) are non-negative for all time, provided that all the $x_{\alpha}(0) \geq 0$ and $s_{\alpha} \geqslant 0$. For this, we need an approximation to the $x_{\alpha}$ valid over a sufficiently short interval of time. Fix $\varepsilon>0$, let $I=[0, \varepsilon]$, and rewrite (3) as an integral system

$$
x_{\alpha}(t)=\int_{0}^{t}\left(-\frac{1}{2} x_{\alpha}^{2}+\sum_{\beta} g_{3 \alpha} x_{3}-c_{\alpha} x_{\alpha}\right)+u_{\alpha}(t) .
$$

for all $\alpha \in V t \in I$, where $u_{\alpha}(t)=x_{\alpha}(0)+\int_{0}^{t} s_{\alpha}$. and $c_{\alpha}=\sum_{, 3} g_{\alpha \beta}$. Define the sequence $u_{\alpha}^{(n)}$ recursively by $u_{\alpha}^{(0)}=u_{\alpha}$ and $u_{\alpha}^{(n+1)}=\int_{0}^{t} u_{\alpha}^{(n)}$ for $n \geqslant 0$. Finally, set

$$
Z_{\alpha}=u_{\alpha}^{(0)}+\sum_{p . t(p)=\alpha} g_{p} u_{o(p)}^{(\ell(p))}
$$


$Z_{\alpha}$ will be shown to be the most significant term in $x_{\alpha}$ as $t \rightarrow 0^{+}$. We begin by proving that the above series is uniformly and absolutely convergent, so in particular $Z_{\alpha}$ is welldefined. In our problem, $u_{\alpha}^{(0)} \geq 0$ for all $\alpha$, so the same is true of all the $u_{\alpha}^{(n)}$. In addition, we have

$$
u_{\alpha}^{(n+r)} \leqslant u_{\alpha}^{(n)} \frac{t^{r}}{r !}
$$

for all $r, n \geqslant 0$. For $n=0$, apply induction on $r$, the case $r=0$ being trivial. If (8) is true for some $r \geqslant 1$ and $n=0$, then $u_{\alpha}^{(r+1)}=\int_{0}^{t} u_{\alpha}^{(r)} \leqslant \int_{0}^{t} u_{\alpha}^{(0)}(\tau) \frac{\tau^{r}}{r !} d \tau$. The definition of $u_{\alpha}^{(0)}$ as the integral of a non-negative function implies that it is a non-decreasing function on $[0, t]$, so the last integral can be majorized by replacing $u_{\alpha}^{(0)}(\tau)$ by $u_{\alpha}^{(0)}(t)$, whence the inequality for $r+1$ follows by integration. Now (8) may be established by induction on $n+r$, using $u_{\alpha}^{(n+r+1)}=\int_{0}^{t} u_{\alpha}^{(n+r)} \leqslant \int_{0}^{t} u_{\alpha}^{(n)}(\tau) \frac{\tau^{r}}{r !} d \tau$, and replacing $u_{\alpha}^{(n)}(\tau)$ by its maximum value $u_{\alpha}^{(n)}(t)$.

Suppose a path $p$ can be obtained by attaching a simple cycle $C$ to a path $q$. Then, $g_{p}=g_{C} g_{q}$ and $\ell(p)=\ell(C)+\ell(q)$, so $g_{p} u_{o(p)}^{(\ell(p))}=g_{C} g_{q} u_{o(q)}^{(\ell(q)+\ell(C))}$, and (8) implies that

$$
g_{p} u_{o(p)}^{(\ell(p))} \leqslant g_{q} u_{o(q)}^{(\ell(q))} \frac{t^{\ell(C)}}{\ell(C) !} .
$$

Now every path can be obtained by attaching a number of simple cycles to a simple path (see Sec. 3), so the series (7) for $Z_{\alpha}$ is obviously majorized by

$$
\left[1+\sum_{j \in J} g_{p_{j}} u_{p_{j}}^{\left(\ell\left(p_{j}\right)\right)}\right] \prod_{C}\left(1-\frac{t^{\ell(C)}}{\ell(C) !}\right)^{-1},
$$

where $\left\{p_{j}: j \in J\right\}$ is the set of simple paths in $\Gamma$ that are not cycles, and the product is over all the distinct simple cycles of $\Gamma$ (if any). The M-test may clearly be applied with the choice of a sufficiently small value of $t$ in the last formula.

Absolute convergence implies that the series for $Z_{\alpha}$ may be re-written in the form

$$
\sum_{p, t(p)=\alpha} g_{p} u_{o(p)}^{(\ell(p))}=\sum_{\beta \alpha \in E} g_{\beta \alpha}\left[\sum_{q, t(q)=\beta, \ell(q) \geqslant 1} g_{q} u_{o(q)}^{(\ell(q)+1)}+u_{\beta}^{(1)}\right],
$$

because any path $p$ of length at least 2 terminating in $\alpha$ consists of an initial part $q$ followed by an edge $\beta \alpha$. By uniform convergence, the inner sum is simply

$$
\sum_{q, t(q)=\beta, \ell(q) \geqslant 1}\left[g_{\beta \alpha} g_{q} u_{o(q)}^{(\ell(q)+1)}+u_{\beta}^{(1)}\right]=\int_{0}^{t}\left[\sum_{q, t(q)=\beta, \ell(q) \geqslant 1} g_{\beta \alpha} g_{q} u_{o(q)}^{(\ell(q))}+u_{\beta}^{(0)}\right]=\int_{0}^{t} Z_{\beta} .
$$

In other words,

$$
Z_{\alpha}=u_{\alpha}+\int_{0}^{t} \sum_{\beta} g_{\beta \alpha} Z_{\beta}
$$

Subtracting this from (6), we find that

$$
x_{\alpha}-Z_{\alpha}=\int_{0}^{t} \sum_{\beta \alpha} g_{\beta \alpha}\left(x_{\beta}-Z_{\beta}\right)+\int_{0}^{t}\left(-\frac{1}{2} x_{\alpha}^{2}-c_{\alpha} x_{\alpha}\right) .
$$


Let $R_{\alpha}=\left|x_{\alpha}-Z_{\alpha}\right|$. Taking absolute values in (9), we obtain

$$
R_{\alpha} \leqslant \int_{0}^{t} \sum_{3 \alpha} g_{3 \alpha} R_{\beta}+\int_{0}^{t}\left|\left(-\frac{1}{2} x_{\alpha}^{2}-c_{\alpha} x_{\alpha}\right)\right|
$$

on $I$. For a function $f=\left(f_{\alpha}(t)\right)_{\alpha \in V}$, define $w_{f}(t)=\max \left\{\left|f_{\alpha}(t)\right|: \alpha \in V\right\}$. Our aim is to prove that there exists a constant $B$ such that. for all sufficiently small $t>0$, the function $R=\left(R_{\alpha}(t)\right)$ satisfies

$$
u_{R}(t) \leqslant B \int_{0}^{t} u^{\prime} z .
$$

To this end, let $d=\max \left\{\sum_{\beta} g_{\beta \alpha}: \alpha \in V\right\}$ and $c \geqslant \sup \left\{c_{\alpha}+\frac{1}{2}\left|x_{\alpha}(t)\right|: \alpha \in V . t \in I\right\}$. Then $\int_{0}^{t}\left|\left(-\frac{1}{2} x_{\alpha}^{2}-c_{\alpha} x_{\alpha}\right)\right| \leqslant c \int_{0}^{t}\left|x_{\alpha}\right| \leqslant \int_{0}^{t} w_{X}$. so (10) immediately leads to $R_{\alpha} \leqslant$ $d \int_{0}^{t} w_{R}+c \int_{0}^{t} w_{X}$. whence

$$
w_{R}(t) \leqslant g(t)+d \int_{0}^{t} w_{R} .
$$

where $g(t)=c \int_{0}^{t} w_{X}$. By Gronwall's Lemma, (12) implies that $w_{R}(t) \leqslant$ $g(t)+d \int_{0}^{t} e^{d(t-s)} g(s) d s$. Now $g$ is a positive increasing function. and so attains its maximum value on $[0 . t]$ at $t$ itsolf, so $w_{R}(t) \leqslant g(t)+d \int_{0}^{t} e^{d(t-t)} g(t)$. That is,

$$
w_{R}(t) \leqslant c(1+d t) \int_{0}^{t} w_{X}
$$

on $I$. Write $A=c(1+d \varepsilon)$. so (13) implies that $\left|x_{\alpha}-Z_{\alpha}\right| \leqslant A \int_{0}^{t} w_{X}$, whence $\left|x_{\alpha}\right| \leqslant$ $\left|Z_{\alpha}\right|+A \int_{0}^{t} w_{X} \leqslant w_{Z}+A \int_{0}^{t} w_{X}^{\prime}$, so $w_{X}^{\prime} \leqslant w_{Z}+A \int_{0}^{t} w_{X}$. A second application of Gronwall's Lemma shows that $w_{X} \leqslant w_{Z}+\int_{0}^{t} e^{A(t-s)} w_{Z}(s) d s$. Integrate both sides and argue as before to estimate the second integral to obtain $\int_{0}^{t} w_{X} \leqslant(1+A t) \int_{0}^{t} w_{R}$, which on substitution into (13) yields (11). with $B=A(1+A \varepsilon)$.

LEMIA 4.1. If $x_{\Omega}(0) \geqslant 0$ and $s_{\alpha}(t) \geqslant 0$ for all $t \geqslant 0$ and all $\alpha$, then $x_{\alpha}(t) \geqslant 0$ for all $t \geqslant 0$.

Proof. Let $t_{0}=\sup \left\{\tau: \tau \geqslant 0\right.$ and every $x_{\alpha} \geqslant 0$ on $\left.[0, \tau]\right\}$. Suppose $t_{0}$ is finite. Then every $x_{\alpha}\left(t_{0}\right) \geqslant 0$ by continuity. Shift the origin of time to $t_{0}$. By assumption, all the $u_{\alpha} \geq 0$. so all the $Z_{\alpha} \geqslant 0$, and then (11) implies that all the $x_{\alpha} \geqslant 0$ for some distance to the right of $t_{0}=0$. This contradicts the definition of $t_{0}$, as required.

Next, we need to prove the analogues of $2.4-2.7$ of [7] in this more general context. Some proofs consist of no more than a judicious addition of subscripts to the proofs in that paper, while others require a different approach.

The assumption that the $s_{\alpha, k}$ be non-negative, and hence locally integrable, implies that the system (4) has a unique solution on any interval $\left[t_{0}, t_{0}+\varepsilon\right]$, such that for all $k \geqslant 1$ we have

$$
y_{\alpha, k}(t)=e^{-\mu_{*}(t)}\left[y_{\alpha, k}\left(t_{0}\right)\right.
$$




$$
\left.+\int_{t_{0}}^{t} e^{\mu_{\alpha}(\tau)}\left(\frac{1}{2} \sum_{i+j=k} y_{\alpha, i}(\tau) y_{\alpha, j}(\tau)+s_{\alpha, k}(\tau)+\sum_{\beta} g_{\beta \alpha} y_{\beta, k}(\tau)\right) d \tau\right]
$$

where

$$
\mu_{\alpha}(t):=\int_{t_{0}}^{t} x_{\alpha}(\tau) d \tau
$$

LEMMA 4.2. In the situation of (2), assume that $y_{\alpha, k}\left(t_{0}\right) \geq 0$ and $\sum_{k=1}^{\infty} y_{\alpha, k}\left(t_{0}\right)<\infty$ for all $k$ and $\alpha$. Then there exists $t_{1}>t_{0}$ such that $\sum_{k=1}^{\infty} y_{\alpha, k}(t)$ converges uniformly on $\left[t_{0}, t_{1}\right]$.

Proof. Let $\varepsilon>0$ be fixed. Define the following:

$$
\begin{aligned}
& y_{\alpha}\left(t_{0}\right):=\sum_{k=1}^{\infty} y_{\alpha, k}\left(t_{0}\right), \quad m_{\alpha}(t):=\sup _{t_{0} \leq s \leq t} e^{-\mu_{\alpha}(s)} \int_{t_{0}}^{s} e^{\mu_{\alpha}(\tau)} d \tau \\
& S_{\alpha}(t):=\sum_{k=1}^{\infty} s_{\alpha, k}(t), \quad d_{\alpha, k}(t):=\sup _{t_{0} \leq s \leq t} y_{\alpha, k}(s) \\
& p_{\alpha, k}(t):=\sup _{t_{0} \leq s \leq t}\left(y_{\alpha, k}\left(t_{0}\right) e^{-\mu_{\alpha}(s)}+e^{-\mu_{\alpha}(s)} \int_{t_{0}}^{s} e^{\mu_{\alpha}(\tau)} s_{\alpha, k}(\tau) d \tau\right) .
\end{aligned}
$$

As $\mu_{\alpha}$ is a non-negative, non-decreasing function (by Lemma 4.1) and $y_{\alpha, k}\left(t_{0}\right)$ and $s_{\alpha, k}$ are non-negative, we obtain an upper bound on $p_{\alpha, k}$ by replacing $e^{-\mu_{\alpha}(s)}$ by 1 and $e^{\mu_{\alpha}(\tau)}$ by $e^{\mu_{\alpha}(t)}$ in the above formula. This yields

$$
p_{\alpha, k}(t) \leq y_{\alpha, k}\left(t_{0}\right)+e^{\mu_{\alpha}(t)} \int_{t_{0}}^{t} s_{\alpha, k}(\tau) d \tau
$$

Summing from 1 to $n$, we obtain

$$
\sum_{k=1}^{n} p_{\alpha, k}(t) \leq \sum_{k=1}^{n} y_{\alpha, k}\left(t_{0}\right)+e^{\mu_{\alpha}(t)} \int_{t_{0}}^{t} \sum_{k=1}^{n} s_{\alpha, k}(\tau) d \tau \leq y_{\alpha}\left(t_{0}\right)+e^{\mu_{\alpha}(t)} \int_{t_{0}}^{t} S_{\alpha}(\tau) d \tau .
$$

Let $n \rightarrow \infty$ to obtain

$$
P_{\alpha}(t)=\sum_{k=1}^{\infty} p_{\alpha, k}(t) \leq y_{\alpha}\left(t_{0}\right)+e^{\mu_{\alpha}(t)} \int_{t_{0}}^{t} S_{\alpha}(\tau) d \tau<\infty .
$$

As the $m_{\alpha}$ are continuous functions of $t>t_{0}$ and tend to zero as $t \rightarrow t_{0}$, we may choose $\varepsilon$ small enough to ensure that certain inequalities are satisfied. First of all, we require that

$$
m_{\alpha}(t) \sum_{\beta} g_{\beta \alpha}<1
$$

for all $\alpha$ and all $t \in I=\left[t_{0}, t_{0}+\varepsilon\right]$. We also require the existence of a constant $R$ such that

$$
R>\max \left\{P_{\alpha}(t): \alpha \in V, t \in I\right\}
$$


and

$$
P_{\alpha}(t)+\frac{1}{2} m_{\alpha}(t) R^{2}+m_{\alpha}(t) \sum_{\beta} g_{\beta \alpha} R \leqslant R,
$$

for all $\alpha \in V, t \in I$. Clearly (15) and (16) are satisfied for all sufficiently small $\varepsilon$, if we choose $R>K=\sup \left\{P_{\alpha}(t): \alpha \in V, t \in\left[t_{0}, t_{0}+\varepsilon_{1}\right]\right\}$, where $\varepsilon_{1}$ is fixed. As for (17), it is equivalent to $R^{2}-2\left(m_{\alpha}^{-1}-\sum_{\beta} g_{\beta \alpha}\right) R+2 P_{\alpha} m_{\alpha}^{-1} \leqslant 0$, with discriminant $\delta(t)=\left(m_{\alpha}^{-1}-\sum_{\beta} g_{\beta \alpha}\right)^{2}-2 P_{\alpha} m_{\alpha}^{-1}$ and largest root $r_{\alpha}(t)=m_{\alpha}^{-1}-\sum_{\beta} g_{\beta \alpha}+\sqrt{\delta(t)}$. As $t \rightarrow t_{0}$, every $m_{\alpha}(t) \rightarrow 0$, whence $\delta(t)$ and $\min \left\{r_{\alpha}(t)\right\}$ both tend to $+\infty$. Choose $\varepsilon<\varepsilon_{1}$ such that every $r_{\alpha}(t)>K$, and then choose $R$ such that $K<R<\inf \left\{r_{\alpha}(t): \alpha \in V, t \in\right.$ $\left.\left[t_{0}, t_{0}+\varepsilon_{1}\right]\right\}$.

On right-hand side of (14), replace the first term by $p_{\alpha, k}(t)$, each of the $y_{*}$ by the corresponding $d_{*}$, and then take the supremum over $[0, t]$ to obtain

$$
d_{\alpha, k}(t) \leqslant p_{\alpha, k}(t)+\frac{m_{\alpha}(t)}{2} \sum_{i+j=k} d_{\alpha, i}(t) d_{\alpha, j}(t)+m_{\alpha}(t) \sum_{\beta} g_{\beta \alpha} d_{\beta, k}(t),
$$

for all $\alpha, k$ and $t \in I$. Define a new sequence $e_{\alpha, k}$ by $e_{\alpha, 1}(t)=d_{\alpha, 1}(t)$ and

$$
e_{\alpha, k}(t)=p_{\alpha, k}(t)+\frac{m_{\alpha}(t)}{2} \sum_{i+j=k} e_{\alpha, i}(t) e_{\alpha, j}(t)+m_{\alpha}(t) \sum_{\beta} g_{\beta \alpha} e_{\beta, k}(t),
$$

for $k \geqslant 2$. Our first task is to prove that $d_{\alpha, k}(t) \leqslant e_{\alpha, k}(t)$ for $\alpha, k, t$. To this end, fix $t$ and write $u_{\alpha, k}=e_{\alpha, k}(t)-d_{\alpha, k}(t)$. Suppose that $u_{\alpha, i} \geqslant 0$ for all $\alpha$ and all $i<k$. Then $d_{\alpha, k}-m_{\alpha} \sum_{\beta} g_{\beta \alpha} d_{\beta, k} \leqslant p_{\alpha, k}+\sum_{i+j=k} d_{\alpha, i} d_{\alpha, j} \leqslant p_{\alpha, k}+\sum_{i+j=k} e_{\alpha, i} e_{\alpha, j}=e_{\alpha, k}-$ $m_{\alpha} \sum_{\beta} g_{\beta \alpha} e_{\beta, k}$. That is,

$$
u_{\alpha . k}-m_{\alpha} \sum_{\beta} g_{\beta \alpha} u_{\beta, k}=v_{\alpha, k} \geqslant 0
$$

for all $\alpha$. In matrix form, this may be written as

$$
\mathrm{A}\left(u_{\alpha, k}\right)=\left(v_{\alpha, k}\right) \text {, }
$$

where $A_{\alpha, \alpha}=1$ and $A_{\alpha, \beta}=-m_{\alpha} \sum_{\beta} g_{\beta \alpha}$ for $\alpha \neq \beta$. Now the $\alpha$-th component of A 1 is $1-m_{\alpha} \sum_{\beta} g_{\beta \alpha}>0$ by (15). By Theorem 3.2, this means that the inverse of $A$ has non-ncgative entries, whence $\left(u_{\alpha, k}\right)=\mathrm{A}^{-1}\left(v_{\alpha, k}\right) \geqslant 0$, which establishes the inductive step in the proof of

$$
d_{\alpha, k}(t) \leqslant e_{\alpha, k}(t)
$$

Our next task is to prove that $\sum_{k=1}^{\infty} e_{\alpha, k}(t)$ is convergent for every $t \in I$. The explicit proof given in [7] no longer works, so we proceed as follows: Fix $t$. Define $e_{\alpha . k}^{(n)}=e_{\alpha, k}^{(n)}(t)$ by $e_{\alpha, k}^{(0)}=0$ and

$$
e_{\alpha, k}^{(n+1)}=p_{\alpha, k}+\frac{m_{\alpha}}{2} \sum_{i+j=k} e_{\alpha, i}^{(n)} e_{\alpha, j}^{(n)}+m_{\alpha} \sum_{\beta} g_{\beta \alpha} e_{\beta, k}^{(n)},
$$

for all $n \geqslant 0, k \geqslant 1$, and $\alpha$. Clearly, $e_{\alpha, k}^{(1)} \geqslant e_{\alpha, k}^{(0)}$, and it is clear from (18) that $e_{\alpha, k}^{(n)} \geqslant$ $e_{\alpha, k}^{(n-1)}$ for all $k$ implies that $e_{\alpha, k}^{(n+1)} \geq e_{\alpha, k}^{(n)}$ for all $k$. In other words, for every $\alpha$ and $k$, the sequence $\left\{e_{\alpha, k}^{(n)}\right\}$ is non-decreasing. Claim that $\sum_{k=1}^{\infty} e_{\alpha . k}^{(n)} \leqslant R$ for all $n, \alpha$, where $R$ is the 
constant satisfying (16) and (17). This is done by induction by $n$, the case $n=1$ being (16). Supposing the inequality to be true for $n$, we have $\sum_{k=1}^{N} e_{\alpha, k}^{(n+1)}=\sum_{k=1}^{N} p_{\alpha, k}+$ $\frac{m_{\alpha}}{2} \sum_{k=1}^{N} \sum_{i+j=k} e_{\alpha . i}^{(n)} e_{\alpha, j}^{(n)}+m_{\alpha} \sum_{\beta} g_{\beta \alpha} \sum_{k=1}^{N} e_{\beta, k}^{(n)} \leqslant P_{\alpha}+\frac{1}{2} m_{\alpha} R^{2}+m_{\alpha} \sum_{\beta} g_{\beta \alpha} R \leqslant R$ by induction and (17). Now let $N \rightarrow \infty$ to obtain $\sum_{k=1}^{\infty} e_{\alpha, k}^{(n+1)} \leqslant R$. In particular, every $e_{\alpha . k}^{(n)} \leqslant R$, so, being non-decreasing, $f_{\alpha, k}=\lim _{n \rightarrow \infty} e_{\alpha . k}^{(n)}$ exists. Letting $n \rightarrow \infty$ in $\sum_{k=1}^{N} e_{\alpha, k}^{(n)} \leqslant R$ yields $\sum_{k=1}^{N} f_{\alpha, k} \leqslant R$, so $\sum_{k=1}^{\infty} f_{\alpha, k} \leqslant R$ for all $\alpha$. But letting $n \rightarrow \infty$ in (18) shows that $f_{\alpha, k}=e_{\alpha, k}$, so finally $\sum_{k=1}^{\infty} e_{\alpha, k}(t) \leqslant R$ for all $\alpha$ and all $t \in I$. Also, it is obvious from the definitions of $e_{\alpha, k}, m_{\alpha}$, and $p_{\alpha, k}$, that $e_{\alpha, k}(t)$ is an increasing function of $t$. Let $t_{1}<t_{0}+\varepsilon$, and let $r_{\alpha, k}=e_{\alpha . k}\left(t_{0}+\varepsilon\right)$. Then

$$
y_{\alpha, k}(t) \leqslant d_{\alpha, k}(t) \leqslant e_{\alpha, k}(t) \leqslant r_{\alpha, k},
$$

and $\sum_{k=1}^{\infty} r_{\alpha, k} \leqslant R$. By the M-test, the series $y_{\alpha}(t)=\sum_{k=1}^{\infty} y_{\alpha, k}(t)$ is uniformly convergent on $\left[t_{0}, t_{1}\right]$, as required.

Lemma 4.3. In the situation of (2), there exists $\varepsilon>0$ such that $y_{\alpha}=x_{\alpha}$ on $[0, \varepsilon]$.

Proof. The proof of Lemma 2.6 in [7] goes through verbatim, if a subscript $\alpha$ is added to everything in sight. At the very end of that proof, one encounters the following system of ODEs satisfied by $z_{\alpha}=y_{\alpha}-x_{\alpha}$ :

$$
z_{\alpha}^{\prime}=z_{\alpha}^{2}+\sum_{\beta} g_{\beta \alpha} z_{\beta}-\sum_{\beta} g_{\alpha \beta} z_{\alpha}
$$

on $[0, \varepsilon]$, subject to the initial conditions $z_{\alpha}(0)=0$ for all $\alpha$. The uniqueness of the solution of this system shows that $z_{\alpha}$ is identically zero on $[0, \varepsilon]$, as claimed.

The next result is the analogue of Lemma 2.7 of [7]. The proof in that paper works with no change but the addition of a subscript $\alpha$.

LEMma 4.4. In the situation of $(2)$, we have $y_{\alpha}(t)=x_{\alpha}(t)$ for all $t \geqslant 0$.

The existence of a solution now follows easily. Lemma 4.4 implies that $x_{\alpha}=\sum_{k=1}^{\infty} y_{\alpha, k}$ for all $t \geqslant 0$. Therefore, (4) is identical to (2), which is another way of saying that the $\left\{y_{\alpha, k}\right\}$ are a solution to $(2)$.

5. Uniqueness. The proof of uniqueness given in [7] also generalizes to the present case. Suppose $\left\{x_{\alpha, k}\right\}$ is a solution to $(2)$ on $[0, T)$. Then the sum $P_{\alpha}(t):=\sum_{k=1}^{\infty} x_{\alpha, k}(t)$ converges for almost all $t \in[0, T)$ and (2) becomes

$$
x_{\alpha, k}^{\prime}=\frac{1}{2} \sum_{i+j=k} x_{\alpha, i} x_{\alpha, j}-P_{\alpha} x_{\alpha, k}+\sum_{\beta} g_{\beta \alpha} x_{\beta, k}-\sum_{\beta} g_{\alpha \beta} x_{\alpha, k}+s_{\alpha, k} .
$$

LEMma 5.1. In the situation of (2), if $\left\{x_{\alpha, k}\right\}$ is any non-negative solution to Eq. (2) on $[0, \infty)$, then the $P_{\alpha}$ are bounded on an interval $[0, a]$ for some $a>0$. 
Proof. Let $P_{\alpha, n}(t)=\sum_{k=1}^{n} x_{\alpha, k}(t)$. Then $x_{\alpha, k}^{\prime} \leqslant \frac{1}{2} \sum_{i+j=k} x_{\alpha, i} x_{\alpha, j}+\sum_{\beta} g_{\beta \alpha} x_{\beta, k}+s_{\alpha, k}$ yiclds

$$
x_{\alpha, k}(t) \leqslant x_{\alpha, k}(0)+\frac{1}{2} \int_{0}^{t} \sum_{i+j=k} x_{\alpha, i} x_{\alpha, j}+\sum_{\beta} g_{\beta \alpha} \int_{0}^{t} x_{\beta, k}+\int_{0}^{t} s_{\alpha, k} .
$$

Choose constants $A_{\alpha} \geqslant P_{\alpha}(0), t_{0} \in(0 . T)$, and $B_{\alpha} \geqslant \int_{0}^{t_{0}} S_{\alpha}$. Summing (19) from 1 to $n$ yiclds

$$
P_{\alpha, n}(t) \leqslant A_{\alpha}+\frac{1}{2} \int_{0}^{t} P_{\alpha . n-1}^{2}+\sum_{\beta} g_{\beta \alpha} \int_{0}^{t} P_{\beta, n}+B_{\alpha} .
$$

We want to find constants $b_{\alpha}$ such that $P_{\alpha, n} \leqslant b_{\alpha}$ for all $t \in[0, a]$. The above inequality suggests that we should arrange to have

$$
A_{\alpha}+\frac{1}{2} \int_{0}^{a} b_{\alpha}^{2} d \tau+\sum_{\beta} g_{\beta \alpha} \int_{0}^{a} b_{\alpha} d \tau+B_{\alpha} \leqslant b_{\alpha},
$$

which simplifies to

$$
a \leqslant \frac{b_{\alpha}-A_{\alpha}-B_{\alpha}}{b_{\alpha}^{2} / 2+b_{\alpha} \sum_{\beta} g_{3 \alpha}} .
$$

This is trivial to satisfy: choose $b_{\alpha}>A_{\alpha}+B_{\alpha}$ for all $\alpha$, and then choose any positive $a$ satisfying the finite number of inequalities in $(20)$.

The proofs of the next two results are identical to those of Lemmas 3.2 and 3.3 of [7]. The second lemma establishes the uniqueness of the non-negative solutions to (2).

Lemma 5.2. Assume that $\left\{x_{\alpha, k}\right\}$ is any non-negative solution to (2). Then $P_{\alpha}=x_{\alpha}$ on $[0, a]$ for some $a>0$.

Lenna 5.3. In the situation of (2), we have $P_{\alpha}=x_{\alpha}$ for all $t \geqslant 0$.

Finally, we prove that the $x_{\alpha}$ are globally bounded.

Lemma 5.4. If all the $s_{\alpha}$ are bounded above on $[0, T)$, then the $x_{\alpha}$ are also bounded above on $[0, T)$.

Proof. For any $t \geqslant 0$, let $f_{\alpha}(t)=\sup \left\{x_{\alpha}(\tau): 0 \leqslant \tau \leqslant t\right\}$. Also, fix constants $K_{\alpha} \geqslant \sup _{t} s_{\alpha}(t)$, and let $k(t)^{2} / 2=\max _{\alpha}\left\{K_{\alpha}+c_{\alpha}^{2} / 2+\sum_{\beta} g_{\beta \alpha} f_{\beta}(t)\right\}$, where $c_{\alpha}=\sum_{\beta} g_{\alpha \beta}$. If $r_{\alpha}=x_{\alpha}+c_{\alpha}$, then it is readily seen that $r_{\alpha}^{\prime}<-\frac{1}{2} r_{\alpha}^{2}+k^{2} / 2$. throughout [0.t] so by the proof of Lemma 4.2 of [7], we have $r_{\alpha}(\tau) \leqslant \max \left\{r_{\alpha}(0), k\right\}$ for all $\tau \in[0 . t]$. Thus $x_{\alpha}(\tau) \leqslant \max \left\{x_{\alpha}(0), k-c_{\alpha}\right\}$, whence

$$
f_{\alpha}(t) \leqslant \max \left\{x_{\alpha}(0), k(t)-c_{\alpha}\right\} .
$$

Let $w=w_{f}(t)=\max _{\alpha}\left\{f_{\alpha}\right\}$. Then $k^{2} / 2 \leqslant K_{\alpha}+c_{\alpha}^{2} / 2+d_{\alpha} w$. where $d_{\alpha}=\sum_{3} g_{3 \alpha}$. Then $c_{\alpha}+f_{\alpha}(t) \leqslant \max \left\{x_{\alpha}(0)+c_{\alpha}, k(t)\right\} \leqslant x_{\alpha}(0)+c_{\alpha}+k(t)$. Using the definition of $k$, this leads to

$$
f_{\alpha}(t) \leqslant x_{\alpha}(0)+\sqrt{K_{\alpha}+c_{\alpha}^{2} / 2+d_{\alpha} w}
$$


whence

$$
w \leqslant x_{\alpha}(0)+\sqrt{K_{\alpha}+c_{\alpha}^{2} / 2+d_{\alpha} w} .
$$

But $w(t)-\sqrt{K_{\alpha}+c_{\alpha}^{2} / 2+d_{\alpha} w(t)} \leqslant x_{\alpha}(0)$ clearly implies that $w(t)$ cannot become arbitrarily large. The result follows because $x_{\alpha} \leqslant f_{\alpha} \leqslant w$.

6. Global Stability. Let $m_{\alpha}=s_{\alpha}(\infty)$. If the $x_{\alpha}(\infty)$ also exist, then (3) implies that $T_{\alpha}=x_{\alpha}(\infty) \geqslant 0$ is a solution of

$$
\frac{1}{2} T_{\alpha}^{2}+\sum_{\beta} g_{\alpha \beta} T_{\alpha}-\sum_{\beta} g_{\beta \alpha} T_{\beta}-m_{\alpha}=0 .
$$

Now suppose that (21) has a non-negative solution (i.e., a solution with all components non-negative). For technical reasons, it is important to be able to concentrate on the positive components of the solution. We begin by showing that this can be achieved by reducing the graph $\Gamma$, if necessary, as follows.

Let $\left(\ell_{\alpha}\right)$ be a non-negative solution of (21). If $\ell_{\alpha}=0$ for some vertex $\alpha$, then (21) shows that $\sum_{\beta} g_{\beta \alpha} \ell_{\beta}+m_{\alpha}=0$, and everything being non-negative, it follows that $m_{\alpha}=0$ and $\ell_{\beta}=0$ for every edge $\beta \alpha$. But this obviously implies that $L_{o(p)}=M_{o(p)}=0$ for every path $p$ terminating in $\alpha$. In other words, $M_{\delta}=0$ for every vertex $\delta$ in the legitimate subgraph ${ }_{\alpha} \Gamma$ of $\Gamma$.

Conversely, suppose that $m_{\alpha}=0$ for every vertex $\alpha$ in a legitimate subgraph $\Delta$ of $\Gamma$. We show that $\ell_{\alpha}=0$ for every vertex of $\Delta$ as well. To this end, note that for such vertices $(21)$ reads

$$
\frac{1}{2} \ell_{\alpha}^{2}+\sum_{\beta} g_{\alpha \beta} \ell_{\alpha}-\sum_{\beta} g_{\beta \alpha} \ell_{\beta}=0,
$$

where all the $\beta$ occurring in the second sum also belong to $\Delta$. Summing (22) over all $\alpha \in V(\Delta)$ yields

$$
\frac{1}{2} \sum_{\alpha \in \Delta} \ell_{\alpha}^{2}+\sum_{\alpha} \sum_{\beta \alpha \in E(\Gamma) \backslash E(\Delta)} g_{\alpha \beta} \ell_{\alpha}=0 .
$$

As all the $\ell_{\alpha} \geqslant 0$, we must indeed have $\ell_{\alpha}=0$ for all $\alpha \in \Delta$, as claimed. The upshot is the following.

Lemma 6.1. Let $\left(\ell_{\alpha}\right)$ be a non-negative solution to (21), and let $\alpha \in V$. Then $\ell_{\alpha}=0$ if and only if $\alpha$ belongs to a legitimate subgraph $\Delta$ of $\Gamma$ such that $M_{\delta}=0$ for every $\delta \in \Delta$. In this case, $\ell_{\delta}=0$ for every vertex of $\Delta$.

Say a subgraph $\Delta$ is null if $\Delta$ is legitimate and $M_{\delta}=0$ for every $\delta \in \Delta$. The previous lemma implies that every $\ell_{\alpha}>0$ if $\Gamma$ has no null subgraphs. On the other hand, suppose that at least one null subgraph is present in $\Gamma$. Clearly every null subgraph is contained in a subgraph of $\Gamma$ that is maximal with respect to being null, so let $\Delta_{1}, \ldots, \Delta_{m}$ be the distinct maximal null subgraphs of $\Gamma$. If $\cup_{i=1}^{m} V\left(\Delta_{i}\right)=V(\Gamma)$, then $\ell_{\alpha}=0$ for every vertex $\alpha$ of $\Gamma$, and there is nothing further to be said. In the contrary case, define a subgraph $\Gamma_{0}$ of $\Gamma$ as follows: Let $V\left(\Gamma_{0}\right)=V(\Gamma) \backslash \cup_{i=1}^{m} V\left(\Delta_{i}\right)$, and let $E\left(\Gamma_{0}\right)$ consist of those edges (if any) of $\Gamma$ that are not incident with any of the $\Delta_{i}$. 
Suppose $\alpha$ is a vertex in $\Gamma_{0}$. Then $\ell_{\alpha} \neq 0$, for otherwise $\alpha$ would belong to a null subgraph, and hence to one of the maximal null subgraphs $\Delta_{i}$. Consider (21) for such a vertex. In the first sum in (21), none of the $\beta$ can belong to $\cup_{i=1}^{m} V\left(\Delta_{i}\right)$, for the existence of an edge $\alpha \rightarrow \beta \in \Delta_{i}$ implies that $\alpha \in \Delta_{i}$ because $\Delta_{i}$ is legitimate. In the second sum, any term involving some $\beta \in \Delta_{i}$ can be ignored because $\ell_{\beta}=0$. In other words, (21) reduces to

$$
\frac{1}{2} \ell_{\alpha}^{2}+\sum_{\alpha \beta \in E\left(\Gamma_{0}\right)} g_{\alpha \beta} \ell_{\alpha}-\sum_{\beta \alpha \in E\left(\Gamma_{0}\right)} g_{\beta \alpha} \ell_{\beta}-m_{\alpha}=0,
$$

and $\ell_{\alpha}>0$ for all $\alpha \in \Gamma_{0}$. This shows that, in discussing the stability of the solutions of (2), we may confine ourselves to the case where $\Gamma$ has no null subgraphs, in which case (21) has at least one strictly positive solution.

We show below (Theorem 6.3) that the system (21) has at most one non-negative solution, and if it does have such a solution, then the $x_{\alpha}(\infty)$ exist for all $\alpha$. Moreover, all the $x_{\alpha, k}(\infty)$ also exist if the $s_{\alpha, k}(\infty)$ exist, and can be computed quite easily. The next result is the main tool used in the proofs.

THEOREM 6.2. Let $\varepsilon>0$ be a scalar and $\underline{u}>0$ a constant vector. Assume that the vector function $\underline{y}=\left(y_{\alpha}(t)\right)$ satisfies the differential inequality $\underline{y}^{\prime} \leqslant \mathrm{N} \underline{y}+\varepsilon \underline{u}$ for all sufficiently large $t$, where the matrix $\mathrm{N}$ is such that

$$
\mathrm{N}_{\alpha \beta} \geqslant 0 \quad \forall \alpha \neq \beta, \quad \underline{1} \mathrm{~N}<0 .
$$

If $\underline{y}$ is bounded for all sufficiently large $t$, then $\underline{y}(\infty)$ exists and $\underline{y}(\infty) \leqslant-\varepsilon N^{-1} \underline{u}$.

Proof. We may clearly shift the time origin so that the above properties hold for all $t \geqslant 0$ (this is for ease of notation only). We begin by proving that

$$
\underline{y}(t) \leqslant e^{N t}\left(\underline{y}(0)+\varepsilon N^{-1} \underline{u}\right)-\varepsilon N^{-1} \underline{u},
$$

for all $t \geqslant 0$. To do this, let $\underline{z}(t)=e^{N t}\left(\underline{y}(0)+\varepsilon N^{-1} \underline{u}\right)-\varepsilon N^{-1} \underline{u}$ and $\underline{w}=\underline{z}-\underline{y}$, so we want to show that $\underline{w} \geqslant 0$ for all $t$. Of course $\underline{z}(0)=\underline{y}(0)$ and $\underline{z}^{\prime}=\mathrm{N} \underline{z}+\varepsilon \underline{u}$, so $\underline{w}(0)=\underline{0}$, and $\underline{w}$ satisfies the differential inequality

$$
\underline{w}^{\prime} \geqslant N \underline{w} .
$$

Let $v_{\alpha}(t)=e^{-N_{\alpha \alpha} t} w_{\alpha}(t)$ and $G_{\alpha \beta}(t)=N_{\alpha \beta} e^{\left(N_{\beta \beta \beta}-N_{\alpha \alpha}\right) t}>0$ for all $\alpha, \beta$. Then (24) may be written as $w_{\alpha}^{\prime}-N_{\alpha \alpha} w_{\alpha}=\sum_{\beta} N_{\alpha \beta} w_{\beta}$, which is equivalent to $v_{\alpha}^{\prime} \geqslant \sum_{\beta} G_{\alpha \beta} v_{\beta}$. As $\underline{v}(0)=\underline{w}(0)=0$, this yields

$$
v_{\alpha}(t) \geqslant \sum_{\beta} \int_{0}^{t} G_{\alpha \beta}(s) v_{\beta}(s) d s
$$

for all $\alpha$. Define the linear operator $T$ by $T(\underline{f})_{\alpha}=\sum_{\beta} \int_{0}^{t} G_{\alpha \beta} f_{\beta}$ for all $\underline{f}=\left(f_{\alpha}\right)$. Then $T$ is an order-preserving operator (i.e., if $f \geq g$, then $T(f) \geq T(g)$; clear because all the $\left.G_{\alpha \beta}>0\right)$. Also, having fixed a finite $t$, all high enough powers of $T$ are contractions (c.f. [3], p. 14 or [1], Volume I, pp. 125-128). Moreover, (25) is simply $\underline{v} \geq T(\underline{v})$. By Theorem 1.6.3 of [3], $\underline{v}$ is greater than or equal (in the partial order on vectors) to the 
unique fixed point of $T$, which is obviously $\underline{0}$. The definition of $\underline{v}$ implies that $\underline{w} \geqslant \underline{0}$ as well, so (23) is established. Next, we show that for all $\alpha$,

$$
\limsup _{t \rightarrow \infty} y_{\alpha} \leqslant-\varepsilon\left(\mathbf{N}^{-1} \underline{u}\right)_{\alpha}
$$

Indeed, Theorem 3.2 applies to the transpose of $-\mathrm{N}$, since $-\mathrm{N}^{T} \underline{1}>\underline{0}$ by assumption. Therefore, every eigenvalue of $\mathrm{N}$ has negative real part, so $e^{\mathrm{N} t} \rightarrow 0$ as $t \rightarrow \infty$. This and (23) imply that for every $\delta>0$, there exists $t_{\delta}$ such that $y_{\alpha}(t)<\delta-\varepsilon N^{-1} \underline{u}$ for all $t>t_{\delta}$ and all $\alpha$. Thus $\lim \sup y_{\alpha} \leqslant \delta-\varepsilon \mathrm{N}^{-1} \underline{u}$, and (26) follows by letting $\delta \rightarrow 0$.

By Theorem 3.2 again, there exists a vector $\underline{d}>\underline{0}$ such that $\mathrm{N} \underline{d}<\underline{0}$. Let $a>0$ be a constant such that

$$
a>\max _{\alpha}\left\{-\varepsilon\left(\mathrm{N}^{-1} \underline{u}\right)_{\alpha} / d_{\alpha},-\varepsilon u_{\alpha} /(\mathrm{N} \underline{d})_{\alpha}\right\} .
$$

In vector form, we have arranged to have $a \mathrm{~N} \underline{d}+\varepsilon \underline{u}<\underline{0}$ and $-a \underline{d}-\varepsilon \mathrm{N}^{-1} \underline{u}<\underline{0}$. Let $\underline{y}=\underline{f}+a \underline{d}$. Substitution into the inequality for $\underline{y}$ leads to $\underline{f}^{\prime} \leqslant \mathrm{N} \underline{f}+a \mathrm{~N} \underline{d}+\varepsilon \underline{u}<\mathrm{N} \underline{f}$, which in component form reads:

$$
f_{\alpha}^{\prime}<\left(N_{\alpha \alpha}\right) f_{\alpha}+\sum_{\beta} N_{\alpha \beta} f_{\beta}
$$

for all $\alpha$. Now $\lim \sup f_{\alpha}=\limsup y_{\alpha}-a v_{\alpha} \leqslant-\varepsilon\left(N^{-1} \underline{u}\right)_{\alpha}-a v_{\alpha}<0$ by the choice of $a$ again, so $f_{\alpha}(t)<0$ for all $\alpha$ and all sufficiently large $t$. Equation (27) then yields

$$
f_{\alpha}^{\prime}-\left(N_{\alpha \alpha}\right) f_{\alpha}<\sum_{\beta} N_{\alpha \beta} f_{\beta} \leqslant 0 .
$$

In other words, the function $g_{\alpha}(t)=e^{-N_{\alpha \alpha} t} f_{\alpha}(t)$ satisfies $g_{\alpha}^{\prime} \leqslant 0$, so $g_{\alpha}$ is non-increasing, and hence so is $f_{\alpha}(t)=e^{N_{\alpha \alpha} t} g_{\alpha}(t)$, since $N_{\alpha \alpha}<0$. As $f=y-a \underline{v}$ is bounded by assumption, $\underline{f}(\infty)$ exists, and hence so does $\underline{y}(\infty)$. The inequality for $\underline{y}(\infty)$ is then simply (26).

The next result indicates the alternatives for system (2): The system either has no local equilibrium points in the positive octant, or it has a global attractor there.

Theorem 6.3. Assume that the $s_{\alpha}(\infty)=m_{\alpha}$ exist for all $\alpha$. Then (21) has at most one non-negative solution. If a solution exists, then $x_{\alpha}(\infty)$ exists for all $\alpha$, and is equal to the solution.

Proof. In view of the earlier comments, we may assume that the system (21) has solutions $>0$. Let $\underline{\ell}=\left(\ell_{\alpha}\right)>0$ be any such solution of (21). It is then trivial to verify that $y_{\alpha}=x_{\alpha}-\ell_{\alpha}$ satisfies

$$
\underline{y}^{\prime} \leqslant \mathrm{N} \underline{y}+\left(s_{\alpha}-m_{\alpha}\right)_{\alpha},
$$

where $N_{\alpha \beta}=g_{\beta \alpha}$ if $\alpha \neq \beta$, and $N_{\alpha \alpha}=-\ell_{\alpha}-\sum_{\beta} g_{\alpha \beta}$. Fix $\varepsilon>0$. Now $s_{\alpha}(t) \rightarrow m_{\alpha}$ as $t \rightarrow \infty$ for every $\alpha$, so $s_{\alpha}-m_{\alpha}<\varepsilon$ for all $\alpha$ and all sufficiently large $t$. By Theorem 6.2, $y(\infty)$ exists, and hence so does $X(\infty)$. Moreover, $y(\infty) \leqslant-\varepsilon N^{-1} \underline{1}$. Letting $\varepsilon \rightarrow 0$, we obtain $\underline{y}(\infty) \leqslant \underline{0}$, so $\underline{x}(\infty) \leqslant \underline{\ell}$. 
As for the uniqueness of $\underline{\ell}$, assume that Eq. (21) also has a solution $\underline{\ell}>0$. If $X(0)=\underline{\ell}$, then $\underline{x}(t)=\underline{\hat{\ell}}$ for all $t$, whence $\underline{\hat{\ell}}=X(x) \leqslant \underline{\ell}$. But interchanging the roles of $\underline{\hat{\ell}}$ and $\underline{\ell}$ shows that $\underline{\ell} \leqslant \underline{\ell}$. Thus $\underline{\ell}=\underline{\ell}$, so the solution is unique.

Finally, we turn to the $x_{\alpha, k}$.

Theorem 6.4. Assume that the $s_{\alpha, k}(\infty)=m_{\alpha . k}$ exist for all $\alpha$ and $k$. Then the $x_{\alpha, k}(\infty)=\ell_{\alpha, k}$ also exist for all $\alpha$ and $k$. and are given by Eq. (29) below.

Proof. Write (4) in the form

$$
x_{\alpha, k}^{\prime}=\frac{1}{2} \sum_{i+j=k} x_{\alpha, i} x_{\alpha, j}-x_{\alpha} x_{\alpha, k}+\sum_{\beta} g_{3 \alpha} x_{3, k}-\sum_{\beta} g_{\alpha, 3} x_{\alpha, k}+s_{\alpha, k} .
$$

The $x_{\alpha, k}(\infty)$, if they exist, must satisfy the system

$$
\left(\underline{\mathrm{P}} \underline{\ell_{k}}\right)_{\alpha}=\frac{1}{2} \sum_{i+j=k} \ell_{\alpha, i} \ell_{\alpha, j}+m_{\alpha, k}
$$

where for $k \geqslant 1$, the column vector $\underline{\ell_{k}}$ (respectively $\underline{m_{k}}$ ) has entries $\ell_{\alpha, k}=x_{\alpha, k}(\infty)$ (resp. $\left.m_{\alpha, k}\right)$, and $\mathrm{P}=\operatorname{diag}\left(\ell_{\alpha}\right)+\mathrm{H}$ has entries

$$
P_{\alpha, \alpha}=\ell_{\alpha}+\sum_{\beta} g_{\alpha \beta}, \quad P_{\alpha . \beta}=-g_{\beta \alpha} \text { for all } \alpha \neq \beta
$$

Observe that $1 \mathrm{P}$ is the vector consisting of the $\ell_{\alpha}>0$, so by Theorem $3.2, \mathrm{P}$ is invertible and its inverse has non-negative entries. Thus, (29) can be recursively solved for the $\underline{\ell_{k}}$, starting with $\underline{\ell_{1}}=\mathrm{P}^{-1} \underline{m_{1}}$.

Now let $\underline{\ell_{k}}$ denote the unique solutions of (29), and set $x_{\alpha, k}=\ell_{\alpha, k}+z_{\alpha, k}$ and $x_{\alpha}=$ $\ell_{\alpha}+y_{\alpha}$. Substitution into (28) eventually leads to

$$
\begin{aligned}
z_{\alpha, k}^{\prime}=\frac{1}{2} \sum_{i+j=k} z_{\alpha, i} z_{\alpha, j} & +\sum_{i+j=k} \ell_{\alpha, i} z_{\alpha, j}+\sum_{\beta} g_{\beta \alpha} z_{\beta, k} \\
& -\left(\ell_{\alpha}+\sum_{\beta} g_{\alpha \beta}\right) z_{\alpha, k}+\ell_{\alpha, k} y_{\alpha}-y_{\alpha} z_{\alpha, k}+s_{\alpha, k}-m_{\alpha, k} .
\end{aligned}
$$

We prove that $\underline{z_{k}}(t) \rightarrow \underline{0}$ as $t \rightarrow \infty$ by induction on $k$. When $k=1$, Eq. (31) simplifies to

$$
z_{\alpha, 1}^{\prime}=\sum_{\beta} g_{\beta \alpha} z_{\beta, 1}-\left(\ell_{\alpha}+\sum_{\beta} g_{\alpha \beta}\right) z_{\alpha .1}+\ell_{\alpha .1} y_{\alpha}-y_{\alpha} z_{\alpha .1}+s_{\alpha, 1}-m_{\alpha .1} .
$$

We know that $y_{\alpha} \rightarrow 0$ and $s_{\alpha, 1} \rightarrow m_{\alpha, 1}$ as $t \rightarrow 0$, so given any $\varepsilon>0$, we have

$$
z_{\alpha, 1}^{\prime} \leqslant \sum_{\beta} g_{\beta \alpha} z_{\beta, 1}-\left(\ell_{\alpha}+\sum_{\beta} g_{\alpha \beta}\right) z_{\alpha, 1}+\varepsilon
$$

for all large enough $t$. By Theorem 6.2 , the $z_{\alpha .1}(\infty)$ exist. Letting $t \rightarrow \infty$ on both sides of (32), we obtain

$$
0=\sum_{\beta} g_{\beta \alpha} z_{\beta, 1}(\infty)-\left(\ell_{\alpha}+\sum_{\beta} g_{\alpha \beta}\right) z_{\alpha, 1}(\infty)
$$


That is, $\mathrm{P} \underline{z_{1}}(\infty)=\underline{0}$. As $\mathrm{P}$ is invertible, we find that $\underline{z_{1}}(\infty)=\underline{0}$. In general, if $\underline{z_{i}}(\infty)=\underline{0}$ for all $i<k$, then for all large enough $t$, Eq. (31) leads to

$$
z_{\alpha . k}^{\prime} \leqslant \sum_{\beta} g_{\beta \alpha} z_{\beta, k}-\left(\ell_{\alpha}+\sum_{\beta} g_{\alpha \beta}\right) z_{\alpha, k}+\varepsilon,
$$

as the other terms have limit zero as $t \rightarrow \infty$, and so can be approximated from above by any fixed $\varepsilon$. The proof that $\underline{z_{k}}(\infty)=\underline{0}$ now proceeds exactly as for $\underline{z}_{1}$. Finally, having proved that all the $\underline{z_{k}}(t) \rightarrow \underline{0}$ as $t \rightarrow \infty$, it follows that $x_{\alpha, k}(\infty)=m_{\alpha, k}$ for all $\alpha$ and $k$, as required.

7. Conservation of Mass. Assume that all the $s_{\alpha, k}=0$. Let $\mu_{\alpha}(t)=\sum_{k=1}^{\infty} k x_{\alpha, k}(t)$ and $\underline{\mu}(t)=\left(\mu_{\alpha}(t)\right)_{\alpha}$. Multiply (2) by $k$ and formally sum to obtain $\underline{\mu}^{\prime}=\mathrm{H} \underline{\mu}$, whence $\underline{\mu}(t)=e^{\mathrm{H} t} \underline{\mu}(0)$ as in (5). (This may be justified rigorously in the usual way, e.g., by using generating functions as in [6]. This is routine and is left to the reader.)

In particular, $\underline{1} \underline{\mu}(t)=\underline{1} \sum_{i=1}^{\infty} \mathrm{H}^{i} t^{i} / i ! \underline{\mu}(0)=\underline{1} \underline{\mu}(0)$. In other words,

$$
\sum_{\alpha} \mu_{\alpha}(t)=\sum_{\alpha} \mu_{\alpha}(0)
$$

for all $t$. This is, of course, conservation of total mass. As already pointed out in Sec. 2, complex eigenvalues of $\mathrm{H}$ can lead to oscillatory behaviour in the components of $\underline{\mu}$. It is worth pointing out, however, that such behaviour can only occur if $\Gamma$ has at least one cycle. More precisely, if $\Gamma$ has no (directed) cycles, then $\mathrm{H}$ has triangular form relative to some suitable ordering of the vertices. As $\mathrm{H}=-\operatorname{diag}\left(c_{\alpha}\right)+\mathrm{G}^{T}$, it is sufficient to prove the result for $\mathrm{G}$.

Suppose $\Gamma$ is a graph with no directed cycles. It is then well-known (and trivial to prove) that $\Gamma$ has at least one initial vertex $\alpha$, i.e., a vertex which is not the terminal vertex of any edge of $\Gamma$. Assign levels to the vertices of $\Gamma$ as follows: The level $t(\alpha)$ of a vertex $\alpha$ is the maximum length of a simple path ending in $\alpha$ (so the level of every initial vertex is zero). This is easily seen to be well-defined and finite. Moreover, let $e=\beta \alpha$ be an edge in $\Gamma$. If $p$ is a simple path of maximal length ending in $\beta$, then pe is a path ending in $\alpha$, so $t(\alpha) \geqslant t(\beta)+1>t(\beta)$.

Arrange the vertices of $\Gamma$ in order of increasing level. If $\alpha$ comes before $\beta$ in this ordering, then $t(\alpha) \leqslant t(\beta)$, so by the above argument there is no edge from $\beta$ to $\alpha$. This means that the matrix of $\mathrm{G}$ only has zero entries below the main diagonal, as claimed.

8. Data. In this section we present the complete set of data for the graphs in Sec. 2 . For $g=g_{\max }$, the results were

\begin{tabular}{|l||l|l|l|l|l|l|l|l|}
\hline & $\mathcal{P}_{3, b}$ & $\mathcal{P}_{5, a}$ & $\mathcal{H}_{2,2}$ & $\mathcal{H}_{3,2}$ & $\mathcal{H}_{4,2}$ & $\mathcal{A}_{2}$ & $\mathcal{A}_{3}$ & $\mathcal{A}_{6}$ \\
\hline \hline$\rho_{4}$ & 0.324 & 0.186 & 0.186 & 0.166 & 0.156 & 0.252 & 0.235 & 0.244 \\
\hline$\rho_{4}^{*}$ & 0.108 & 0.037 & 0.037 & 0.024 & 0.017 & 0.126 & 0.078 & 0.041 \\
\hline
\end{tabular}

For $g=0.2$, the numbers were as follows: 


\begin{tabular}{|l||l|l|l|l|l|l|l|l|}
\hline & $\mathcal{P}_{3, b}$ & $\mathcal{P}_{5, a}$ & $\mathcal{H}_{2,2}$ & $\mathcal{H}_{3,2}$ & $\mathcal{H}_{4,2}$ & $\mathcal{A}_{2}$ & $\mathcal{A}_{3}$ & $\mathcal{A}_{6}$ \\
\hline \hline$\rho_{4}$ & 0.359 & 0.286 & 0.323 & 0.295 & 0.272 & 0.337 & 0.364 & 0.417 \\
\hline$\rho_{4}^{*}$ & 0.12 & 0.057 & 0.065 & 0.042 & 0.030 & 0.169 & 0.121 & 0.07 \\
\hline
\end{tabular}

\section{REFERENCES}

[1] F. R. Gantmacher, The Theory of Matrices, Chelsea, 1959

[2] M. Gimenez, M. Schlamp, and A. Clausse, Analysis of the spatial distribution of aerosol dispersions, Annals of Nuclear Energy 22, 17-28 (1995)

[3] E. Hille, Ordinary Differential Equations In the Complex Domain, Dover, 1997

[4] A. Kuciauskas, Tracking troposphoric aerosols across the Pacific Ocean, Naval Postgraduate School Report, 1999

[5] M. H. Lee, On the validity of the coagulation equation and the nature of runaway growth, Icarus $143,74-86$ (2000)

[6] M. Shirvani and H. J. Van Roessel, The mass-conserving solutions of Smoluchowski's coagulation equation: The general bilinear kernel, Z. angew. Math. Phys. 43, 526-535 (1992)

[7] M. Shirvani and H. J. Van Roessel, Existence and uniqueness of solutions of Smoluchowski's coagulation equation with source terms, Quarterly of Applied Mathematics LX(1), 183-194 (2002)

[8] H. Wielandt, Topics in the Analytic Theory of Matrices, Department of Mathematics, University of Wisconsin-Madison, 1967 